\title{
Effectiveness of the cyclic administration of dienogest in a case of pathological disappearance of intestinal endometriosis
}

This article was published in the following Dove Press journal:

International Journal of Women's Health

15 July 2013

Number of times this article has been viewed

\author{
Ryo Tamura \\ Ikunosuke Tsuneki \\ Toru Yanase \\ Department of Obstetrics and \\ Gynecology, Niigata City General \\ Hospital, Niigata, Japan
}

\begin{abstract}
We have reported good control of atypical genital bleeding when using a cyclic administration of dienogest (repeated 4-week cycles, each consisting of the administration of $2 \mathrm{mg} /$ day of dienogest for 3 weeks, followed by 1 week of drug withdrawal) in patients with endometriosis. Herein, we report the effectiveness of the long-term cyclic administration (22 months) of dienogest in a case of pathological disappearance of intestinal endometriosis diagnosed by endoscopy and histology of the lower gastrointestinal tract. There is no recurrent sign after 16 months of the treatment being stopped. Atypical genital bleeding during treatment was 3-5 days a month in each cycle. Compliance was good, so we could continue the therapy. The long-term cyclic administration of dienogest in patients with intestinal endometriosis may have significant merit.
\end{abstract}

Keyword: atypical genital bleeding, dysmenorrhea, drug compliance, endoscopy, histology

\section{Introduction}

The predominant symptoms of intestinal endometriosis are melena during menstruation, defecation pain, and anal pain. The diagnosis and treatment of this disease are often difficult.

Dienogest can be administered for longer periods and has less adverse drug reactions than conventional drugs such as gonadotropin-releasing hormone agonists. However, we have experienced cases where medicine compliance decreases due to atypical genital bleeding during treatment for long periods. In contrast, we have reported good control of atypical genital bleeding when using a cyclic administration of dienogest.

Herein, we report the case of a patient who was diagnosed with intestinal endometriosis by endoscopy and histology of the lower gastrointestinal tract and was successfully treated by the long-term cyclic administration of dienogest.

\section{Case report Patient}

The patient was a 45 -year-old woman with no previous pregnancies.

\section{History of present illness}

The patient experienced an ongoing exacerbation of menstrual cramps around July 2008 and felt lumbago and lower abdominal pain while not menstruating. She visited a physician in January 2009. An endometriotic cyst that was about $5 \mathrm{~cm}$ in size was observed on the right side of the uterus by transvaginal ultrasonography. She was 
referred to our department for detailed examination and treatment, and she visited us in January of the same year.

\section{Findings at initial visit}

A $55 \mathrm{~mm} \times 48 \mathrm{~mm}$ ovarian tumor on the right side and adenomyosis lesion of the back wall of the uterus was observed by transvaginal ultrasonography. A blood test showed that cancer antigen (CA) 125 levels were as high as $297.9 \mathrm{U} / \mathrm{mL}$. In addition, a $45 \mathrm{~mm} \times 40 \mathrm{~mm}$ endometriotic cyst was observed on the right side using magnetic resonance imaging (Figure 1, upper image).

\section{Therapeutic course I}

The patient selected conservative therapy. We first decided to treat the dysmenorrhea symptoms with keishi-bukuryo-gan, a Kampo medicine, and oral analgesics. However, in February of the same year, the patient had diarrhea and melena on the first day of menstruation and visited an internist. We performed a lower gastrointestinal endoscopy in April of the same year.

Figure 2 (upper images) show the endoscopic findings of the lower gastrointestinal tract. Redness and hemorrhagic upheaved lesions were observed on the anal side of the sigmoid colon and the upper part of the rectum, and therefore, a biopsy of these lesions was performed. Figure 3 (upper images) shows the histopathological findings. Ectopic endometriotic tissues were observed in both mucosae of the sigmoid colon and the upper part of the rectum.

\section{Therapeutic course 2}

Although the biopsy results showed intestinal endometriotic lesions, we continued with the same treatment, mainly consisting of pain control because neither serious diarrhea nor defecation disorder was observed and because the patient did not want to undergo surgical treatment or endocrine treatment. However, the lower abdominal pain and melena continued, and she visited us and asked for endocrine treatment. We then initiated the cyclic administration of dienogest (repeated 4-week cycles, each consisting of the administration of $2 \mathrm{mg}$ /day of dienogest for 3 weeks, followed by 1 week of drug withdrawal) on the second day of menstruation in August of the same year. We started treatment after obtaining her informed consent. We considered the effectiveness and any problematic adverse effects of the cyclic administration of dienogest and continued this treatment for as long as possible.

The patient's clinical signs and symptoms changed after the initiation of the cyclic administration of dienogest.
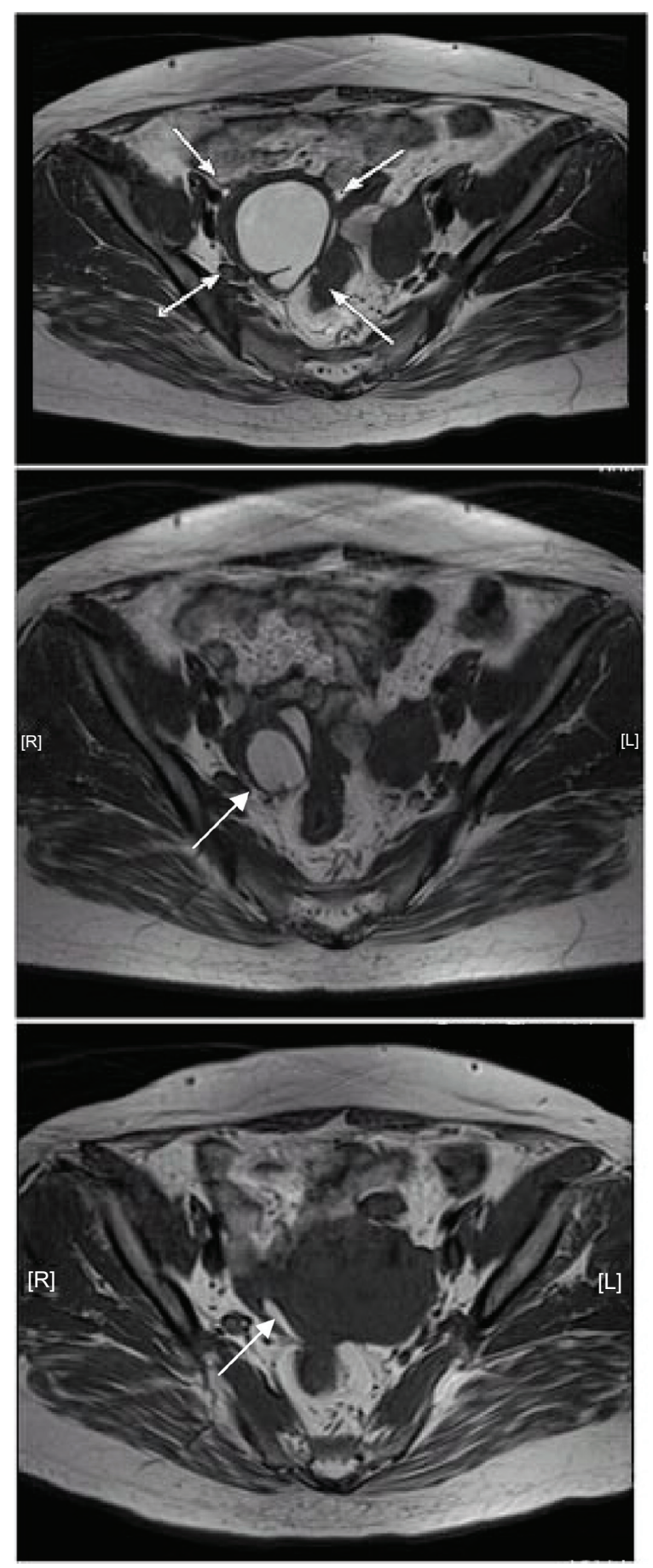

Figure I Magnetic resonance imaging showing changes after the administration of dienogest. Upper image, in January 2009; middle image, in April 2010; lower image, in June 20I I. The size of the endometriotic cyst markedly decreased from $45 \mathrm{~mm} \times 40 \mathrm{~mm}$ before treatment to $27 \mathrm{~mm} \times 20 \mathrm{~mm}$ in April 2010 and to $24 \mathrm{~mm} \times 5 \mathrm{~mm}$ in June $201 \mathrm{I}$. Note: The arrows point to ovarian cysts.

Abbreviations: L, left; R, right.

The lower abdominal pain was markedly improved (the value on the visual analog scale of lower abdominal pain was decreased from 10 to 2 or 3 ) in the second month of treatment with dienogest, and melena decreased to once or twice a month in the next 6 months of administration and 
Anal side of the sigmoid colon
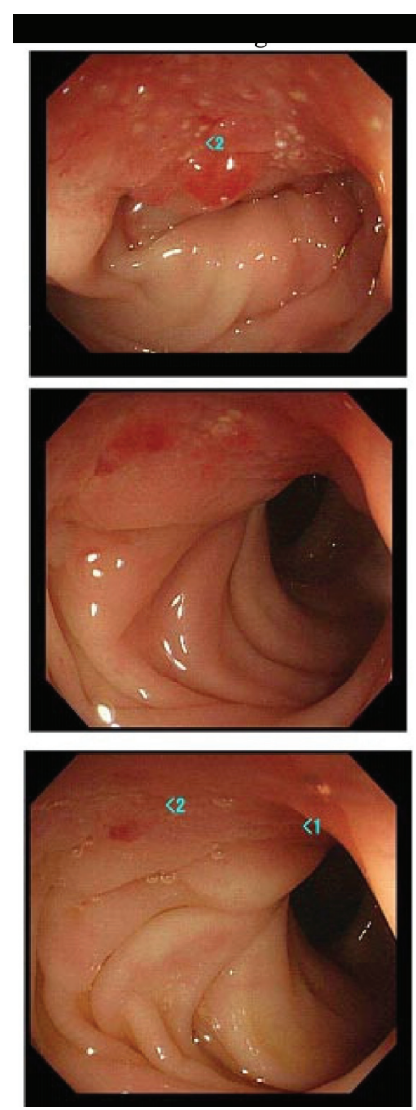

Figure 2 Changes in lower gastrointestinal endoscopic images. Upper images, taken in 2009; middle images, taken in 2010; lower images, taken in 2011 .

Note: $1-3$ is the area biopsy was performed.

thereafter. CA125 levels decreased gradually to $38 \mathrm{U} / \mathrm{mL}$. The size of the endometriotic cyst had markedly decreased to $24 \mathrm{~mm} \times 5 \mathrm{~mm}$ in June 2011 (Figure 1, lower image). A lower gastrointestinal endoscopy that was performed in April 2010 revealed that the lesions in both the sigmoid clone and the upper part of the rectum had markedly decreased (Figure 2, middle images). When a lower gastrointestinal endoscopy was performed again in June 2011, the endometriotic lesions were not observed endoscopically (Figure 2, lower images). When a second biopsy of the polypoid part was performed during the lower gastrointestinal endoscopy in June 2011, no evidence of ectopic endometriotic tissue was found. Therefore, we concluded that the intestinal endometriosis lesions had disappeared (Figure 3, lower images). Atypical genital bleeding during treatment was 3-5 days a month in each cycle. Minor atypical genital bleeding was observed during treatment. Compliance was good, so we could continue the therapy.

We continued the treatment until April 2012. There was no evidence of ectopic endometriotic tissue in a third biopsy of the polypoid part performed in May 2012. There were no endometriotic cyst and extant smaller adenomyosis
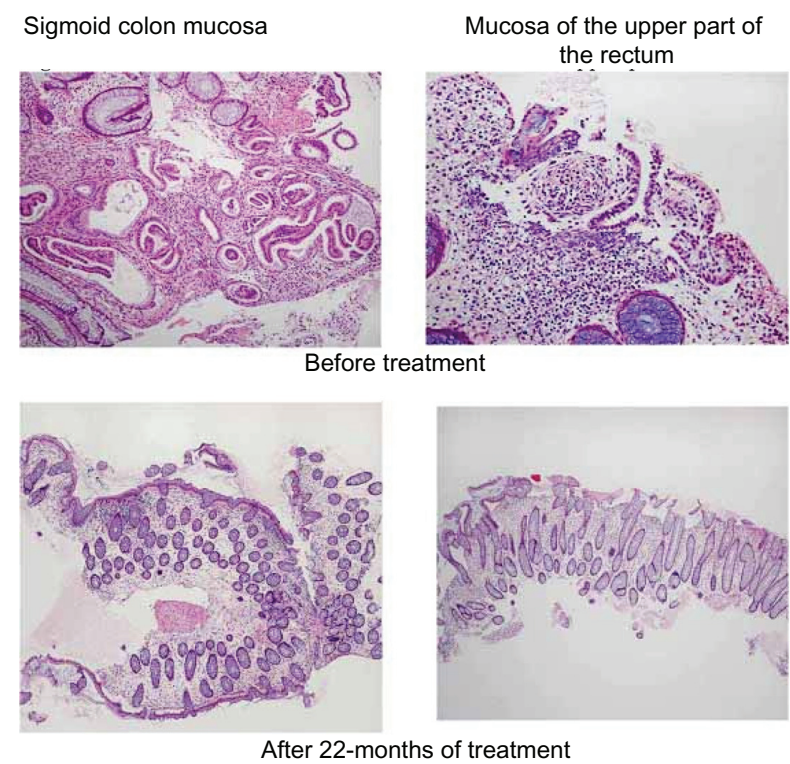

Figure 3 Changes in histopathological findings. Upper pictures, before treatment; lower pictures, after 22-months of treatment.

lesion shown by magnetic resonance imaging performed in May 2012. Dual-energy X-ray absorption performed in May 2012 was normal. Lipid blood test was also normal. There were no signs of recurrence, such as lumbago, lower abdominal pain, melena, and elevated level of CA125 after 16 months of the treatment being stopped.

\section{Discussion}

Intestinal endometriosis is found in about $5 \%-37 \%$ of all cases of endometriosis. The most common sites for lesions are the rectum and the sigmoid colon, but they are also found in the small intestine, cecum, and appendix. ${ }^{1}$ Its diagnosis is often difficult despite the presence of severe symptoms during menstruation. Surgery is difficult to perform and involves the risk of a number of complications, such as injury to the ureter or the intestine. Although improvements in treatment outcomes are expected due to endocrine therapies, gonadotropin releasing hormone $(\mathrm{GnRH})$ agonists and danazol are not suitable treatments because their durations of administration are limited. Long-term pharmacotherapy with low-dose pills may be problematic due to adverse events, such as hepatic function disorders, and thrombosis.

Dienogest is believed to induce both an antiproliferative effect on endometriotic cells and a reduction of estrogen levels due to the inhibition of ovarian function. ${ }^{2-5}$ It has been reported that, while dienogest shows a similar level of efficacy as GnRH agonists, it causes less menopausal symptoms or bone loss. ${ }^{5,6}$ Dienogest at $2 \mathrm{mg}$ a day is recommended as the optimal dose for provision of efficacy 
and safety in endometriosis studies. ${ }^{7}$ The most problematic adverse event related to dienogest treatment is atypical genital bleeding during treatment. Ways to cope with this problem, including an improved regimen for dienogest use, ie, cyclic administration, ${ }^{8}$ and a treatment regimen in which a GnRH agonist is administered before dienogest, ${ }^{9}$ have been reported. The cyclic administration of dienogest reported by Yanase is a method of reducing atypical genital bleeding excluding withdrawal bleeding (menstruation-like bleeding) in drug withdrawal. Yanase reported that the majority of patients had withdrawal bleeding, and cyclic administration of dienogest decreased atypical genital bleeding to 2-5 days a month in each cycle compared with 10-18 days during the continuous administration, without getting worse. The serum estradiol level for the cyclical administration decreased more gradually and remained a little higher (about $50 \mathrm{pg} / \mathrm{mL}$, finally) than the continuous administration. We expected effectiveness of the cyclic administration of dienogest in the therapeutic window range and could reduce problematic adverse effects such as hot flushes. ${ }^{8}$

Harada et $\mathrm{al}^{10}$ reported that the administration of dienogest in five patients with ectopic endometriosis (sigmoid colon, four patients; rectum, 1 patient) for more than 6 months swiftly improved the symptoms in all patients and that the sizes of the affected lesions were decreased after 10-11 months of administration. In the present case, the effectiveness of dienogest was seen during the 6 months of administration, and the disappearance of intestinal endometriosis was confirmed in 16 months of the treatment. The long-term administration of dienogest using the cyclic administration method in patients with intestinal endometriosis that is hard to manage may have significant merit in that it not only exhibits sufficient efficacy, but also is thought to contribute to the improvement of compliance due to its few adverse events and to the low cost.

\section{Disclosure}

The authors report no conflicts of interest in this work.

\section{References}

1. Chiou YY, Pitman MB, Hahn PF, et al. Rare benign and malignant appendiceal lesions: spectrum of computed tomography findings with pathologic correlation. J Comput Assist Tomogr. 2003;27(3):297-306.

2. Fu L, Osuga Y, Morimoto C, et al. Dienogest inhibits BrdU up-take with G0/G1 arrest in cultured endometriotic stormal cells. Fertil Steril. 2008;89(Suppl 5):1344-1347.

3. Oettel M, Carol W, Elger W, et al. A 19-norprogestin without 17 $\alpha$-ethinyl group II: dienogest from a pharmacodynamic point of view. Drugs Today. 1995;312(7):517-536.

4. Sasagawa S, Shimizu Y, Nagaoka T, Tokado H, Imada K, Mizuguchi K. Dienogest, a selective progestin, reduces plasma estradiol level through induction of apoptosis of granulose cells in the ovarian dominant follicle without follicle-stimulating hormone suppression in monkeys. J Endocrinol Invest. 2008;31:636-641.

5. Harada T, Momoeda M, Taketani Y, et al. Dienogest is as effective as intranasal buserelin acetate for the relief of pain symptoms associated with endometriosis - a randomized, double-blind, multicenter, controlled trial. Fertil Steril. 2009;91(3):675-681.

6. Strowitzki T, Marr J, Gerlinger C, et al. Dienogest is as effective as leuprolide acetate in treating the painful symptoms of endometriosis: a 24-week, randomized, multicentre, open-label trial. Hum Reprod. 2010;25:633-641.

7. Köhler G, Faustmann TA, Gerlinger CH, et al. A dose-ranging study to determine the efficacy and safety of 1,2, and $4 \mathrm{mg}$ of dienogest daily for endometriosis. Int J Gynecol Obstet. 2010;108:21-25.

8. Yanase T. [Alleviation of atypical genital bleeding by dienogest treatment: a clinical report]. Hormone Frontier in Gynecology. 2009;16(4):68-73. Japanese.

9. Kitawaki J, Kusuki I, Yamanaka K, et al. Maintenance therapy with dienogest following gonadotropin-releasing hormone agonist treatment for endometriosis-associated pelvic pain. Eur J Obstet Gynecol Reprod Biol. 2011;157(2):212-216.

10. Harada M, Osuga Y, Izumi G, et al. Dienogest, a new conservative strategy for extragenital endometriosis: a pilot study. Gynecol Endocrinol. Sep 2011;27(9):717-720.
International Journal of Women's Health

\section{Publish your work in this journal}

The International Journal of Women's Health is an international, peerreviewed open-access journal publishing original research, reports, editorials, reviews and commentaries on all aspects of women's healthcare including gynecology, obstetrics, and breast cancer. The manuscript management system is completely online and includes

\section{Dovepress}

a very quick and fair peer-review system, which is all easy to use. Visit http://www.dovepress.com/testimonials.php to read real quotes from published authors. 